\title{
Cohort-Comparison of Thoracic Endovascular Aortic Repair with Open Thoracic Aortic Repair Using Modern End-Organ Preservation Strategies
}

\author{
Dean J. Arnaoutakis, MDa, George J. Arnaoutakis, MDa, Christopher J. Abularrage, MD ${ }^{\mathrm{a}}$, \\ Robert J. Beaulieu, MDa ${ }^{\mathrm{a}}$, Ashish S. Shah, MD $^{\mathrm{b}}$, Duke E. Cameron, MD $^{\mathrm{b}}$, and James H. Black \\ III, MDa
}

aDivision of Vascular and Endovascular Therapy, The Johns Hopkins Hospital, Baltimore, Maryland 'bivision of Cardiac Surgery, The Johns Hopkins Hospital, Baltimore, Maryland

\begin{abstract}
Introduction-Pivotal trials showed that thoracic endovascular aortic repair (TEVAR) has improved outcomes compared to open surgery for treating descending thoracic aortic aneurysms. However, those trials included historical open controls in which modern end-organ preservation strategies were not routinely employed. To create a more level assessment, we compared our outcomes of elective TEVAR to modern open thoracic aortic repair (OTAR) controls.
\end{abstract}

\begin{abstract}
Materials and Methods-A retrospective review of thoracic aortic aneurysm patients undergoing TEVAR was compared to a contemporaneous cohort of OTAR patients. Partial bypass or hypothermic circulatory arrest (HCA) was used in all OTAR patients. Cerebrospinal fluid (CSF) drain placement was attempted in all patients. Preoperative characteristics, operative variables, and outcomes were recorded, and the Kaplan-Meier method was used for survival estimates.
\end{abstract}

Results-The main outcome was mortality. Secondary outcomes included postoperative spinal cord ischemia (SCI) or stroke, and any persistent neurologic deficit 30 days following the operation. During the study period, 62 patients underwent TEVAR and 56 underwent OTAR with median follow-up of 23.7 months and 36.4 months, respectively. No difference existed between the TEVAR and OTAR with respect to overall neurologic complications ( $8.1 \%$ vs $12.5 \%$; $=.55)$ as well as any residual neurologic deficit at 30 days ( $0 \%$ vs $5.4 \%, \mathrm{P}=.10)$. TEVAR patients had fewer complications including pneumonia $(\mathrm{P}=.02)$, rebleeding $(\mathrm{P}=.02)$, and acute kidney injury ( $\mathrm{P}=.001)$. There was no difference in 30 -day mortality (1.6\% vs $8.9 \%, \mathrm{P}=.10), 1$-year mortality ( $12.2 \%$ vs $14 \%, \mathrm{P}=.80)$, or 5 -year mortality ( $53.9 \%$ vs $44 \%, \mathrm{P}=.48)$ between TEVAR and OTAR, respectively.

Conclusions-TEVAR continues to show improved perioperative outcomes with a trend towards decreased 30-day mortality and fewer major adverse events compared to OTAR. However,

Correspondence: James H. Black III, MD, Division of Vascular Surgery and Endovascular Therapy, The Johns Hopkins Hospital, Halsted 668, 600 N Wolfe Street, Baltimore, MD 21287, USA, Tel: 410-955-1708, Fax: 410-614-2079, jhblack@jhmi.edu.

Declaration of Conflicting Interests:

The authors declared no conflicts of interest with respect to the authorship and/or publication of this article. 
with the routine use of end-organ preservation strategies during OTAR, neurologic deficits, particularly SCI, can be safely reduced to comparable levels with those of TEVAR and 1-year allcause mortality rates are similar between the groups. These OTAR results may serve as a benchmark as TEVAR is increasingly applied for other aortic pathologies, such as chronic dissection, wherein long-term efficacy is not proven.

\section{Keywords}

Thoracic Aneurysm Repair; TEVAR; Cardiac Bypass; CSF drain; RIFLE

\section{Introduction}

Traditional open thoracic aortic repair (OTAR) for descending thoracic aortic aneurysms (DTAA) carries substantial morbidity including neurologic, pulmonary, renal, and bleeding complications. Mortality rates following open repair have only recently improved and now approach $10 \%$, particularly at high-volume centers of excellence. ${ }^{1,2,3,4}$ Much of the improvement is related to refined strategies that minimize the frequency and severity of endorgan injury and spinal cord ischemia (SCI). These techniques aim to increase visceral and spinal cord blood flow, often via partial left heart bypass (LHB), cerebrospinal fluid (CSF) drainage, and reimplantation of intercostal arteries. Neuroprotective strategies also minimize SCI through hypothermia and epidural cooling. 5,6

Thoracic endovascular aortic repair (TEVAR) has been increasingly applied to a variety of thoracic aortic pathology. ${ }^{7}$ Much of the support for TEVAR relates to the reported decrease in perioperative morbidity and mortality and improvement in quality of life compared to the traditional open repair. $8,9,10$ The original Food and Drug Administration Investigational Device Exemption (FDA IDE) studies that led to approval of the currently available thoracic aortic stent graft devices showed favorable results for TEVAR with marked reductions in mortality, stroke, and permanent paralysis. ${ }^{11,12,13}$

However, these pivotal trials included historical open controls in which modern end-organ preservation strategies were not routinely employed. In an effort to create a more level and contemporary assessment, we compared our outcomes of elective TEVAR to modern open thoracic aortic repair (OTAR) controls.

\section{Materials and Methods}

\section{Patient Data}

We used a prospectively collected database to perform a retrospective review of DTAA patients undergoing TEVAR from March 2002 through February 2013. A contemporaneous cohort of OTAR patients from the same time period was included for comparison. Demographic variables including age, gender, and race as well as cardiovascular comorbidities were collected from the medical chart. Data on the patient's aortic pathology and presentation were recorded. All patients whose dissection or aneurysm extended to the abdominal aorta (zones 6-11) were excluded. ${ }^{14}$ Additional exclusion criteria included traumatic aortic pathology as well as ruptured presentation. For those undergoing OTAR, 
operative records were reviewed to identify the bypass technique utilized (partial LHB, hypothermic circulatory arrest (HCA)), degree of hypothermia (moderate, circulatory arrest), number of intercostals reimplanted, the level of the proximal and distal anastomosis, and total length of aortic replacement. For those undergoing TEVAR, operative notes, intraoperative angiograms, and postoperative CT angiograms were evaluated to identify the number of devices deployed, stent graft distance from the left subclavian artery (LSA) and the celiac artery, and total length of aortic stent graft coverage. Adjunctive procedures, including CSF drainage and LSA bypass, were attempted in all patients except those patients in emergency settings.

\section{Outcomes}

The main outcome of the study was 1-year all-cause mortality. Secondary outcomes included 30-day mortality, 5-year mortality, and the presence of any neurologic complication, defined as postoperative SCI (paraparesis or paraplegia) or stroke. Neurologic complications were prospectively entered into a database. Additionally, we identified patients who had a persistent neurologic deficit, based on both objective and subjective strength and function scores, at 30 days following the operation. Postoperative complications including acute kidney injury (AKI), myocardial infarction (MI), pneumonia, bleeding, wound infection, and death were identified. Hospital length of stay (LOS), intensive care unit (ICU) LOS, and disposition to a rehabilitation facility were also recorded. The computerized medical record and the Social Security Death Index were queried to obtain long-term follow-up and survival status in order to calculate 1- and 5-year all-cause mortality.

Patients were classified as having AKI according to the RIFLE criteria based on the international consensus definition from the Acute Dialysis Quality Initiative Workgroup. ${ }^{15}$ We defined AKI to include any patient who met criteria for RIFLE-R, -I, or -F. eGFR was calculated using the Modification of Diet in Renal Disease formula. ${ }^{16,17}$

\section{Technique for OTAR and Heart Bypass}

The operative strategy for OTAR using partial LHB at Johns Hopkins Hospital has been described. ${ }^{5}$ The descending thoracic aorta is exposed through a posterolateral thoracotomy and the left inferior pulmonary vein is dissected and controlled. Simultaneously, the left common femoral artery is exposed and an 8-mm Dacron chimney is attached to the vessel in an end-to-side fashion. Proximal and distal control of the aorta is then obtained. Systemic heparin is administered $(100 \mathrm{U} / \mathrm{kg}$ ) and a venous cannula is placed into the left atrium via the pulmonary vein. An arterial cannula is advanced cephalad through the previously placed Dacron chimney along with an indwelling central line for distal perfusion pressure monitoring. The cannulas are connected to a centrifugal pump and flow rates of 3.5 to 4.5 $\mathrm{L} / \mathrm{min}$ are usually required to maintain distal aortic perfusion pressure. The patient is cooled to $33.8^{\circ} \mathrm{C}$ and the aneurysm is then opened longitudinally after cross clamping of the aorta. After appropriate sizing of a 24- to 30-mm Dacron graft for aortic replacement, proximal and distal anastomoses are carried out in the standard fashion. Proximal intercostal arteries are oversewn; however, intercostals distal to T6 are often temporarily occluded and reimplanted into the Dacron graft. The patient is warmed, protamine is administered, cannulas 
are removed, venotomy and arterial chimney sites are repaired, drains are positioned in the left chest, and the thoracotomy incision is closed.

For patients who had previous aortic arch surgery (ie., previous hemiarch repair) and presented with lesions involving the proximal descending thoracic aorta, mobilizing the aorta at the level of the left subclavian artery can be hazardous. In these situations, we selectively employ HCA for repair. Preparation, positioning, and incision are similar to LHB approach. Arterial and venous cannulas are placed in the left common femoral vessels under echocardiographic guidance and cardiopulmonary bypass is initiated after cannulating the left atrium. Profound hypothermia to $18^{\circ} \mathrm{C}$ is achieved by gradual cooling and circulation is arrested. After the distal aortic arch is cross-clamped, the aorta is opened and the proximal anastomosis is constructed as described above followed by restoration of cerebral circulation. The distal anastomosis is fashioned after flushing the native aorta and graft of air and debris and the intercostals are addressed as described above. The patient is warmed, cardiac activity returns, and cannulas are removed.

\section{Endovascular Technique for TEVAR}

The technique for TEVAR at Johns Hopkins has been previously described. ${ }^{18}$ All TEVAR procedures are performed using the Gore TAG (WL Gore \& Associates, Flagstaff, Arizona) endovascular prosthesis. The right femoral artery is isolated through a groin incision and a $22 \mathrm{~F}$ sheath is inserted after systemic heparinization. Percutaneous left transfemoral access is obtained and a $5 \mathrm{~F}$ sheath is inserted. A wire is advanced into the abdominal aorta under fluoroscopy and pigtail catheter is positioned above the aortic bifurcation. Aortic dimensions are delineated by angiography prior to device deployment. Post-deployment angiograms are performed to document placement and absence of endoleak.

\section{CSF Drainage and LSA Bypass}

The approach for CSF drainage and LSA bypass at The Johns Hopkins Hospital has been recently published. ${ }^{18}$ At our institution, CSF drainage is attempted in all patients, except for those in emergent situations, since the catheter permits us to more safely, quickly, and effectively address any neurologic changes that may develop. For patients undergoing CSF drainage, 5F lumbar catheter (Codman, USA) was introduced into the subarachnoid space at the L3 or L4 intervertebral space typically on the day prior to surgery. On the day of TEVAR, the catheter was connected to a pressure transducer and a drainage bag for continuous monitoring. Drainage of CSF was initiated if the CSF pressure exceeded $10 \mathrm{~cm}$ $\mathrm{H}_{2} \mathrm{O}$, with the collection system leveled at the left earlobe. The drain was clamped on postoperative day 1 and removed on postoperative day 2 if the patient had a normal neurologic exam.

Currently, if coverage of the LSA is necessary due to anatomic constraints for proximal device deployment, a carotid-subclavian bypass using a 6- or 8-mm Dacron graft is routinely performed prior to TEVAR in a staged fashion. In our early experience, carotid-subclavian bypass was performed selectively for maintenance of LIMA to LAD coronary bypass. 


\section{Statistical Analysis}

Continuous variables are presented with the mean \pm standard deviation (SD) or with the median and interquartile range (IQR). Categorical variables are displayed as whole numbers and percentages. A comparative analysis of baselines characteristics and postoperative outcomes between TEVAR and OTAR groups was performed. When comparing continuous variables, Student's t-test and Wilcoxon test were used for parametric and nonparametric data, respectively. Fisher's exact test or Chi-square test was used for comparing categorical variables. A univariate analysis estimated predictors of mortaility at 1-year. The KaplanMeier method was used to estimate survival. For statistical analyses, P-values less than .05 (two-tailed) were deemed significant. Hazard ratios (HR) are presented with $95 \%$ confidence intervals (CI). Statistical testing was conducted using STATA 12 software (StataCorp LP, College Station, TX).

\section{Results \\ Demographics and Presentation}

During the study period (2002-2013), 62 patients underwent TEVAR and 56 underwent OTAR. A relatively similar distribution of patients underwent TEVAR $(n=30,48 \%)$ or OTAR ( $n=23,41 \%)$ during the first 6 years of the study. TEVAR patients were significantly older $(\mathrm{P}<.001)$, more likely to suffer from coronary artery disease $(\mathrm{P}=.001)$ and chronic obstructive pulmonary disease $(\mathrm{P}=.017)$, and had worse baseline renal function based on eGFR $(\mathrm{P}=.024)$ compared to OTAR patients. TEVAR patients were more likely to suffer degenerative aneurysmal disease while OTAR patients were more likely to present with chronic dissection. Additional preoperative characteristics are presented in Table I.

\section{Operative Details}

Operative data are shown in Table II. Of the 62 TEVAR patients, 13 (21\%) underwent a staged LSA bypass procedure prior to the endovascular aortic repair due to anticipated coverage of the origin of the LSA. Forty-seven patients $(76 \%)$ had a CSF drain placed preoperatively. Of the 56 OTAR patients, 51 patients $(91 \%)$ had a CSF drain placed preoperatively. All OTAR patients underwent cardiac bypass with $50 \%$ of the cohort requiring $\mathrm{HCA}$.

\section{Outcomes}

The median length of follow-up for the TEVAR and OTAR patients was 23.7 and 36.4 months, respectively. No difference existed between the TEVAR and OTAR with respect to overall neurologic complications $(8.1 \%$ vs $12.5 \%$; $\mathrm{P}=.55)$. Of the 5 patients in the TEVAR group who had a neurologic complication, 3 (4.8\%) developed a stroke and $2(3.2 \%)$ developed SCI. Of the 7 OTAR patients who sustained a neurologic complication, 3 (5.4\%) had a stroke and $4(7.1 \%)$ had signs consistent with SCI. Additional review of the patients with a neurologic complication was undertaken to identify whether they had a persistent neurologic deficit 30 days after the operation. None $(0 \%)$ of the TEVAR patients had a residual neurologic deficit whereas $3(5.4 \%)$ OTAR patients had a persistent deficit at 30 days; this difference, however, did not reach statistical significance $(\mathrm{P}=.10)$. Additional 
outcomes between the groups, including wound infection, rebleeding, MI, and the development of AKI, are reported in Table III. There was no difference $(\mathrm{P}=.80)$ in the 1-year all-cause mortality rate between TEVAR (12.2\%) and OTAR (14\%). Although 30-day mortality was better in the TEVAR group (1.6\%) compared to OTAR (8.9\%), this finding did not reach statistical significance $(\mathrm{P}=.10)$.

Predictors of 1-year survival on univariate analysis included baseline eGFR $(\mathrm{P}=.03)$ and overall neurologic complications ( $\mathrm{P}=.01$ ) (Table IV). Surgical approach (TEVAR vs OTAR) did not significantly affect estimated 5-year survival ( $\mathrm{P}=.51)$ (Figure I).

\section{Discussion}

Open repair of the descending thoracic aorta has been the traditional therapy for degenerative thoracic aortic aneurysms. However, this technique remains a surgical challenge as it is associated with substantial morbidity and mortality, even in centers of excellence. Initially, the "clamp and sew" technique was the mainstay approach and speed was paramount in order to minimize the profound homeostatic disturbances of simple aortic cross clamping and to prevent SCI. During the "clamp and sew" era of the 1970s and 1980s, the persistent SCI rates approached $10 \%$ and the cumulative morbidity exceeded $60 \% .{ }^{19,20,21}$ Given these poor results, adjunctive procedure like partial LHB and CSF drains were introduced in order to preserve end-organ perfusion. These measures have improved outcomes; in most modern series, reported 30-day mortality rates generally approach $10 \%$ with $2 \%$ to $4 \%$ of patients suffering from paraplegia. $1,2,3,4,20,22$ As such, we were compelled to examine our outcomes, particularly neurologic complications, in order to assess the efficacy of utilizing adjunctive end-organ strategies when performing TEVAR or OTAR.

The morbidity of open repair in conjunction with the advancement of endovascular therapy has led to the wide application of TEVAR. The original FDA IDE studies examining TEVAR showed improvements with 30-day mortality rates ranging from $1.9 \%$ to $2.1 \%$ and paraplegia rates ranging from $1.3 \%$ to $3 \%$ compared to the open controls whose mortality rates ranged from $5.7 \%$ to $11.7 \%$ and paraplegia rates ranged from $3.4 \%$ to $14 \% .11,12,13$ Given these encouraging findings, the authors concluded that TEVAR is a safer and effective treatment for descending thoracic aortic aneurysms compared to traditional open repair. However, these pivotal trials included historical open controls in which modern end-organ preservation strategies were not routinely employed, and consequently motivated the examination of our outcomes in this endeavor. In the GORE TAG trial, only 78\% of the open control patients had repairs undertaken on extracorporeal bypass and the CSF drain rate was variable and dependent on the participating institution. ${ }^{11}$ The Medtronic Talent VALOR trial did not provide any details of the historical open controls used in the study. ${ }^{12}$ In the Cook Zenith TX2 trial, $31.4 \%$ of the open control patients had distal aortic perfusion, $34.3 \%$ had some form of hypothermia, and $77.1 \%$ had a CSF drain placed. ${ }^{13}$ In contrast, modern endorgan preservation strategies were routinely employed in our study; $100 \%$ of the OTAR patients underwent cardiac bypass for distal perfusion and $91 \%$ had an adjunctive CSF drain placed. 
A key finding of this study, in contrast to the pivotal trials, is that we found no difference in either overall or persistent neurologic outcomes, including both SCI and stroke, between TEVAR and OTAR. We surmise the adjunctive maneuvers routinely used to maximize spinal cord perfusion during open repair helped reduce our rate of persistent neurologic deficit at 30 days to only $5.4 \%(\mathrm{n}=3)$. In fact, a residual neurologic deficit was attributable to SCI in only $1(1.8 \%)$ of these 3 OTAR patients, a level comparable to that of TEVAR. We demonstrate that the dreaded neurologic sequelae of an open repair can be mitigated through the routine use of adjunctive measures. Thus, our study challenges the conventional wisdom that longer lengths of thoracic aortic coverage or graft replacement are explicit risk factors for paraplegia. Indeed, our strategy of employing end-organ preservation strategies may abrogate the excess physiologic stress of more extensive repairs regardless of surgical approach. To wit, the focus of intercostal preservation, in our view, may be overemphasized; instead, by maintaining adjuncts and higher perfusion pressures, "length of coverage" concerns may be mitigated.

Similar to the reported data in the pivotal FDA IDE trials, our 30-day mortality rate for TEVAR (1.6\%) was decreased compared to OTAR (8.9\%), albeit this did not reach statistical significance in our study. However, an all-cause mortality analysis at 1-year demonstrated a more equal distribution of survivors between the TEVAR (12.2\%) and OTAR (14\%). Thus, our results do not contradict previous reports, as our study reaffirms the safety, limited morbidity, and short-term effectiveness of TEVAR. Furthermore, all-cause mortality at 5 years was similar between the groups suggesting the long-term efficacy of TEVAR.

Notably, half of the OTAR patients underwent cardiopulmonary bypass with HCA. HCA allows for safe construction of the proximal anastomosis but is likely a major driving factor for the 30-day mortality in our OTAR cohort given some disadvantages of HCA including coagulopathy causing difficulty in controlling bleeding, retraction injury to a heparinized lung, cold injury to the lung, and a profound inflammatory response from the bypass circuit. ${ }^{23,24,25}$ This is further reflected by the increased 30-day mortality rate comparing HCA (10.7\%) with partial left heart bypass (7.1\%). In fact, half of the OTAR mortalities in the series were directly related to complications of profound HCA (cardiac failure after rewarming and hemorrhagic stroke). We continue to employ HCA selectively for OTAR, typically in patients with a history of connective tissue disorder and/or prior hemiarch repair, as it allows for the safe construction of the proximal anastomosis in these situations; however, we have modified our practice over the years to minimize these complications. To address the risk of cardiac failure after rewarming, we now routinely use transesophageal echocardiography to observe the left ventricle during the bypass procedure to reduce its filling thereby minimizing the potential for subendocardial ischemia. To address hemorrhagic complications related to the increased fibrinolysis of deep hypothermia, we do not drain CSF while the patient is profoundly hypothermic in order to minimize the pressure and subsequent tearing of dural veins.

Similar to other reports, $21 \%$ of our overall study group developed AKI; this complication occurred more frequently and with more severity in our OTAR cohort compared to the TEVAR group. This finding is likely related to the physiologic effects of profound hypothermia in those undergoing HCA including increased systemic vascular resistance, 
decreased cardiac output, and a left shift in the oxyhemoglobin saturation curve, all of which contribute to decreased oxygen delivery to tissues. ${ }^{35}$ Despite starting with a lower baseline eGFR compared to OTAR patients, only $10 \%$ of the TEVAR cohort sustained AKI, which is lower than other reports.

This previous finding provides some guidance with respect to which patients we offer TEVAR as opposed to OTAR. Patients who present with renal insufficiency and/or chronic aortic dissection are likely better served by undergoing TEVAR as opposed to OTAR. Anatomically, we remain cautious in applying TEVAR to dissection patients as ongoing false lumen perfusion has been a longstanding concern. Furthermore, many chronic dissection patients in our series have connective tissue disorders, for which TEVAR has specific concern (ie., retrograde Type A dissection, stent-induced aortic tears, etc). As previously mentioned, patients with connective tissue disorders and/or prior hemiarch repair are best suited for OTAR under HCA in order to safely construct the proximal anastomosis. In addition, age has become a principal consideration for TEVAR given our clinical experience with the elderly and the prolonged convalescence associated with OTAR.

We believe future studies comparing TEVAR to OTAR should ensure that the OTAR cohort includes only those patients who undergo modern end-organ preservation strategies; otherwise, the comparison is arguably biased towards TEVAR outcomes. For example, Desai and colleagues reviewed their series of patients who had thoracic aortic aneurysms repaired by either TEVAR or OTAR. ${ }^{26}$ Each of the 45 OTAR patients underwent either partial left heart bypass or HCA. The TEVAR cohort had a trend toward decreased 30-day mortality as well as decreased rates of neurologic complications; however, none of these findings reached statistical significance. On long-term follow-up, TEVAR versus open approach did not influence survival, which is similar to what we observed in our study. Consequently, clinical decision making for TEVAR and OTAR should not explicitly focus on paraplegia. Indeed, the management of chronic aortic dissection remains controversial. We believe many of these patients can be treated by either surgical approach with durable outcomes so long as end-organ preservation strategies are routinely utilized.

Limitations of the study include its retrospective design and lack of randomization, thereby subjecting the results to selection and measurement bias. Also, we must stress that the two cohorts are not identical, in that there are more dissection patients in the OTAR group among other differences, which cannot be ignored when interpreting the results of our comparison. The results may not be generalizable as the institutional experience with this demanding disease has driven refinements in techniques, particularly for the selection of OTAR. In addition, this study, which includes a small sample of patients from a single center, may be underpowered to identify differences in the outcome variables, particularly if the postoperative events (SCI, stroke) are rare. Also, the sample size limits our ability to perform more sophisticated statistics, such as a Cox multivariable regression model, and to make more generalizable results. The sample size is even smaller at 5 years which adversely affects our survival estimates of both groups. This limitation may be magnified in the TEVAR group since this cohort is older and had a higher prevalence of coronary artery disease. Finally, the majority of our long-term follow-up is based on the Social Security Index thereby allowing us to comment on all-cause mortality only as opposed to disease- 
specific mortality. This factor in conjunction with not reporting reintervention rates prevents us from making more substantial conclusions regarding long-term outcomes, particularly since TEVAR patients often require additional procedures for endoleak. A follow-up study is needed to address this shortcoming. Cost analysis is an increasingly important driver of treatment strategy; we did not capture cost data for this project, but have previously published in this regard. ${ }^{36} \mathrm{~A}$ follow-up study regarding resource utilization would be interesting since we found that an equal number of patients were discharged home following TEVAR and OTAR.

\section{Conclusions}

TEVAR continues to show improved perioperative outcomes with decreased 30-day mortality and fewer major adverse events compared to OTAR. However, with the routine use of end-organ preservation strategies during OTAR, neurologic deficits, particularly SCI, can be safely reduced to comparable levels with those of TEVAR and 1-year all-cause mortality rates are similar between the groups. These OTAR results may serve as a benchmark as TEVAR is increasingly applied for other aortic pathologies, such as chronic dissection, wherein long-term efficacy is not proven.

\section{Acknowledgments}

Funding:

The authors received no financial support for the research and/or authorship of this article.

\section{References}

1. Coselli JS, LeMaire SA, Conklin LD, Adams GJ. Left heart bypass during descending thoracic aortic aneurysm repair does not reduce the incidence of paraplegia. Ann Thorac Surg. 2004; 77:1298-1303. [PubMed: 15063255]

2. Estrera AL, Rubenstein FS, Miller CC 3rd, Huynh TT, Letsou GV, Safi HJ. Descending thoracic aortic aneurysm: Surgical approach and treatment using the adjuncts cerebrospinal fluid drainage and distal aortic perfusion. Ann Thorac Surg. 2001; 72:481-486. [PubMed: 11515886]

3. Galloway AC, Schwartz DS, Culliford AT, et al. Selective approach to descending thoracic aortic aneurysm repair: A ten-year experience. Ann Thorac Surg. 1996; 62:1152-1157. [PubMed: 8823105]

4. Verdant A, Cosette R, Page A, Baillot R, Dontigny L, Page P. Aneurysms of the descending thoracic aorta: Three hundred sixty-six consecutive cases resected without paraplegia. J Vasc Surg. 1995; 21:385-390. [PubMed: 7877220]

5. Black JH 3rd, Cambria RP. Contemporary results of open surgical repair of descending thoracic aortic aneurysms. Semin Vasc Surg. 2006 Mar; 19(1):11-7. [PubMed: 16533687]

6. Cambria RP, Davison JK, Zannetti S, L'Italien G, Atamian S. Clinical experience with epidural cooling for spinal cord protection during thoracic and thoracoabdominal aneurysm repair. J Vasc Surg. 1997; 25:234-241. [PubMed: 9052558]

7. Cambria RP, Crawford RS, Cho JS, et al. GORE TAG Investigators. A multicenter clinical trial of endovascular stent graft repair of acute catastrophes of the descending thoracic aorta. J Vasc Surg. 2009 Dec; 50(6):1255-64. [PubMed: 19958982]

8. Gopaldas RR, Huh J, Dao TK, et al. Superior nationwide outcomes of endovascular versus open repair for isolated descending thoracic aortic aneurysm in 11,669 patients. J Thorac Cardiovasc Surg. 2010; 140:1001-10. [PubMed: 20951252] 
9. Orandi BJ, Dimick JB, Deeb GM, Patel HJ, Upchurch GR Jr. A population-based analysis of endovascular versus open thoracic aortic aneurysm repair. J Vasc Surg. 2009; 49:1112-6. [PubMed: 19307086]

10. Abraha I, Romagnoli C, Montedori A, Cirocchi R. Thoracic stent graft versus surgery for thoracic aneurysm. Cochrane Database Syst Rev. 2009 Jan.21:1.

11. Bavaria JE, Appoo JJ, Makaroun MS, Verter J, Yu ZF, Mitchell RS. Gore TAG Investigators. Endovascular stent grafting versus open surgical repair of descending thoracic aortic aneurysms in low-risk patients: a multicenter comparative trial. J Thorac Cardiovasc Surg. 2007; 133:369-77. [PubMed: 17258566]

12. Fairman RM, Criado F, Farber M, et al. VALOR Investigators. Pivotal results of the Medtronic Vascular Talent Thoracic Stent Graft System: the VALOR trial. J Vasc Surg. 2008; 48:546-54. [PubMed: 18572352]

13. Matsumura JS, Cambria RP, Dake MD, Moore RD, Svensson LG, Snyder S. TX2 Clinical Trial Investigators. International controlled clinical trial of thoracic endovascular aneurysm repair with the Zenith TX2 endovascular graft: 1-year results. J Vasc Surg. 2008; 47:247-57. [PubMed: 18241743]

14. Fillinger MF, Greenberg RK, McKinsey JF, Chaikof EL. Society for Vascular Surgery Ad Hoc Committee on TEVAR Reporting Standards. Reporting standaards for thoracic endovascular aortic repair (TEVAR). J Vasc Surg. 2010 Oct; 52(4):1022-33. [PubMed: 20888533]

15. Bellomo R, Ronco C, Kellum JA, Mehta RL, Palevsky P. Acute renal failure-definition, outcome measures, animal models, fluid therapy and information technology needs: the Second International Consensus Conference of the Acute Dialysis Quality Initiative (ADQI) Group. Crit Care. 2004; 8:R204-12. [PubMed: 15312219]

16. Stevens LA, Coresh J, Greene T, Levey AS. Assessing kidney function-measured and estimated glomerular filtration rate. N Engl J Med. 2006; 354:2473-83. [PubMed: 16760447]

17. Arnaoutakis GJ, Bihorac A, Martin TD, et al. RIFLE criteria for acute kidney injury in aortic arch surgery. J Thorac Cardiovasc Surg. 2007 Dec; 134(6):1554-60. discussion 1560-1. [PubMed: 18023682]

18. Arnaoutakis DJ, Arnaoutakis GJ, Beaulieu RJ, Abularrage CJ, Lum YW, Black JH. Results of Adjunctive Spinal Drainage and/or Left Subclavian Artery Bypass in Thoracic Endovascular Aortic Repair. Ann Vasc Surg. In press.

19. Livesay JL, Cooley DA, Ventemiglia RA, et al. Surgical experience in descending thoracic aneurysmectomy with and without adjuncts to avoid ischemia. Ann Thorac Surg. 1985; 145:3746. [PubMed: 3966835]

20. Hamerlijnck RP, Rutsaert RR, De Geest R, Brutel de la Riviere A, Defauw JJ, Vermeulen FE. Surgical correction of descending thoracic aortic aneurysms under simple aortic cross-clamping. $\mathrm{J}$ Vasc Surg. 1989; 9:568-573. [PubMed: 2709524]

21. Huynh TT, Miller CC 3rd, Estrera AE, Porat EE, Safi HJ. Thoracoabdominal and descending thoracic aortic aneurysm surgery in patients aged 79 years or older. J Vasc Surg. 2002; 36:469_ 475. [PubMed: 12218969]

22. Black JH 3rd, Cambria RP. Current results of open surgical repair of descending thoracic aortic aneurysms. J Vasc Surg. 2006 Feb; 43(Suppl A):6A-11A.

23. Safi HJ, Miller CC 3rd, Subramaniam MH, et al. Thoracic and thoracoabdominal aortic aneurysm repair using cardiopulmonary bypass, profound hypothermia, and circulatory arrest via left side of the chest incision. J Vasc Surg. 1998 Oct; 28(4):591-8. [PubMed: 9786251]

24. Crawford ES, Coselli JS, Safi HJ. Partial cardiopulmonary bypass, hypothermic circulatory arrest, and posterolateral exposure for thoracic aortic aneurysm operation. J Thorac and Cardiovasc Surg. 1987; 94:824-7. [PubMed: 3682852]

25. Coselli JS, Bozinovski J, Cheung C. Hypothermic circulatory arrest: safety and efficacy in the operative treatment of descending and thoracoabdominal aortic aneurysms. Ann Thorac Surg. 2008; 85:956-964. [PubMed: 18291178]

26. Desai ND, Burtch K, Moser W, et al. Long-term comparison of thoracic endovascular aortic repair (TEVAR) to open surgery for the treatment of thoracic aortic aneurysms. J Thorac Cardiovasc Surg. 2012 Sep; 144(3):604-9. discussion 609-11. [PubMed: 22898503] 
27. Huynh TT, van Eps RG, Miller CC 3rd, et al. Glomerular filtration rate is superior to serum creatinine for prediction of mortality after thoracoabdominal aortic surgery. J Vasc Surg. 2005 Aug; 42(2):206-12. [PubMed: 16102615]

28. Eggebrecht H, Breuckmann F, Martini S, et al. Frequency and outcomes of acute renal failure following thoracic aortic stent-graft placement. Am J Cardiol. 2006 Aug 15; 98(4):458-63. [PubMed: 16893697]

29. Bellomo R, Kellum JA, Ronco C. Defining and classifying acute renal failure: from advocacy to consensus and validation of the RIFLE criteria. Intensive Care Med. 2007 Mar; 33(3):409-13. [PubMed: 17165018]

30. Kuitunen A, Vento A, Suojaranta-Ylinen R, Pettilä V. Acute renal failure after cardiac surgery: evaluation of the RIFLE classification. Ann Thorac Surg. 2006 Feb; 81(2):542-6. [PubMed: 16427848]

31. Arnaoutakis GJ, George TJ, Robinson CW, et al. Severe acute kidney injury according to the RIFLE (risk, injury, failure, loss, end stage) criteria affects mortality in lung transplantation. J Heart Lung Transplant. 2011 Oct; 30(10):1161-8. [PubMed: 21620737]

32. Yue JN, Luo Z, Guo DQ, et al. Evaluation of acute kidney injury as defined by the risk, injury, failure, loss, and end-stage criteria in critically ill patients undergoing abdominal aortic aneurysm repair. Chin Med J (Engl). 2013 Feb; 126(3):431-6. [PubMed: 23422102]

33. Pisimisis GT, Khoynezhad A, Bashir K, Kruse MJ, Donayre CE, White RA. Incidence and risk factors of renal dysfunction after thoracic endovascular aortic repair. J Thorac Cardiovasc Surg. 2010 Dec; 140(6 Suppl):S161-7. [PubMed: 21092786]

34. Piffaretti G, Mariscalco G, Bonardelli S, et al. Predictors and outcomes of acute kidney injury after thoracic aortic endograft repair. J Vasc Surg. 2012 Dec; 56(6):1527-34. [PubMed: 23058721]

35. Bush HL Jr, Hydo LJ, Fischer E, Fantini GA, Silane MF, Barie PS. Hypothermia during elective abdominal aortic aneurysm repair: The high price of avoidable morbidity. J Vasc Surg. 1995; 21:392-402. [PubMed: 7877221]

36. Arnaoutakis GJ, Hundt JA, Shah AS, Cameron DE, Black JH 3rd. Comparative analysis of hospital costs of open and endovascular thoracic aortic repair. Vasc and Endovasc Surg. 2011 Jan; 45(1): $39-45$. 


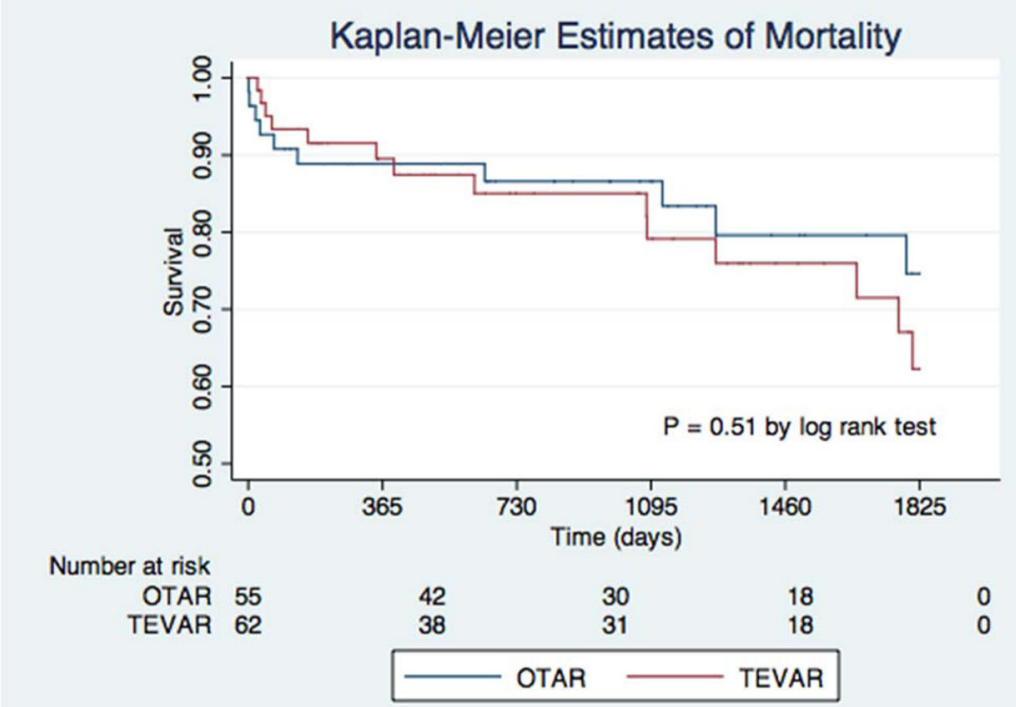

Figure I.

Kaplan-Meier Estimates of 5-year Survival 


\section{Table I}

\section{Preoperative Characteristics}

\begin{tabular}{|c|c|c|c|}
\hline Variables & TEVAR, $n=62$ & OTAR, $n=56$ & P-value \\
\hline Mean age, years (SD) & $67.6(12.9)$ & $56.4(14.3)$ & $<.001$ \\
\hline Male gender, \# $(\%)$ & $33(53)$ & $38(68)$ & .105 \\
\hline Hypertension, \# (\%) & $55(89)$ & $43(77)$ & .085 \\
\hline Smoking history, \# (\%) & $36(58)$ & $20(36)$ & .015 \\
\hline Coronary artery disease, \# (\%) & $21(34)$ & $5(9)$ & .001 \\
\hline Chronic obstructive pulmonary disease, \# (\%) & $16(26)$ & $5(9)$ & .017 \\
\hline Baseline eGFR, $\mathrm{ml} / \mathrm{min} / 1.73 \mathrm{~m}^{2}(\mathrm{SD})$ & $77.15(27.3)$ & $87.91(23.2)$ & .024 \\
\hline Peripheral vascular disease, \# (\%) & $15(24)$ & $6(11)$ & .056 \\
\hline Diabetes mellitus, \# (\%) & $10(16)$ & $6(11)$ & .391 \\
\hline Symptomatic, \# (\%) & $25(40)$ & $30(54)$ & .150 \\
\hline Aortic Pathology & & & $<.001$ \\
\hline Dissection, \# (\%) & $11(18)$ & $35(63)$ & \\
\hline Degenerative aneurysm, \# (\%) & $46(74)$ & $17(30)$ & \\
\hline Pseudoaneurysm, \# (\%) & $5(8)$ & $1(2)$ & \\
\hline Other, \# (\%) & $0(0)$ & $3(5)$ & \\
\hline Mean aneurysm size, cm (SD) & $5.9(1.3)$ & $6.3(1.0)$ & .079 \\
\hline
\end{tabular}

Abbreviations: SD, standard deviation; eGFR, estimated glomerular filtration rate 


\section{Table II}

Operative Details

\begin{tabular}{lc}
\hline Operative Data & \\
\hline TEVAR (n = 62) & $13(21)$ \\
LSA bypass, \# (\%) & $47(76)$ \\
CSF drain, \# (\%) & $23.5(17-30)$ \\
Median length of aortic coverage, cm (IQR) & $2(1-3)$ \\
Median number devices deployed, cm (IQR) & $200(100-300)$ \\
Median blood loss, cc (IQR) & \\
OTAR (n = 56) & $2(4)$ \\
LSA bypass, \# (\%) & $51(91)$ \\
CSF drain, \# (\%) & $28(50)$ \\
Partial LHB, \# (\%) & $28(50)$ \\
Full bypass with HCA, \# (\%) & $18(32)$ \\
Reimplantation of intercostal arteries, \# (\%) & $16.4(13-20)$ \\
Median length of aortic replacement, cm (IQR) & $3200(1375-7000)$ \\
Median blood loss, cc (IQR)
\end{tabular}

Abbreviations: TEVAR, thoracic endovascular aneurysm repair; LSA, left subclavian artery; CSF, cerebrospinal fluid; IQR, interquartile range; OTAR, open thoracic aneurysm repair; LHB, left heart bypass; HCA, hypothermic circulatory arrest 
Table III

\section{Outcomes}

\begin{tabular}{lccc}
\hline Variables & TEVAR, $\mathbf{n = 6 2}$ & OTAR, $\mathbf{n = 5 6}$ & P-value \\
\hline Median length of stay, days (IQR) & $6(3-9)$ & $13(10-20)$ & $<.001$ \\
Median ICU length of stay, days (IQR) & $2(1-3)$ & $4(3-9)$ & $<.001$ \\
Discharge to home, \# (\%) & $52(83.9)$ & $41(73.2)$ & 0.157 \\
Myocardial infarction, \# (\%) & $0(0)$ & $1(1.8)$ & .475 \\
Pneumonia, \# (\%) & $4(6.5)$ & $13(23.2)$ & .016 \\
Rebleeding, \# (\%) & $0(0)$ & $5(8.9)$ & .022 \\
Wound infection, \# (\%) & $2(3.2)$ & $3(5.4)$ & .667 \\
Acute Kidney Injury, \# (\%) ${ }^{a}$ & $6(9.7)$ & $19(34.6)$ & .001 \\
Hemodialysis, \# (\%) & $0(0)$ & $4(6.9)$ & .048 \\
Overall neurologic complication, \# (\%) & $5(8.1)$ & $7(12.5)$ & .546 \\
$\quad$ Spinal cord ischemia, \# (\%) & $2(3.2)$ & $4(7.1)$ & .421 \\
$\quad$ Stroke, \# (\%) & $3(4.8)$ & $3(5.4)$ & .99 \\
Persistent neurologic deficit at 30 days, \# (\%) & $0(0)$ & $3(5.4)$ & .104 \\
30-day mortality, \# (\%) & $1(1.6)$ & $5(8.9)$ & .100 \\
1-year mortality, \# (\%) $b$ & $6(12.2)$ & $7(14.0)$ & .796 \\
5-year mortality, \# (\%) ${ }^{\mathcal{c}}$ & $14(53.9)$ & $11(44.0)$ & .482 \\
\hline
\end{tabular}

Abbreviations: IQR, interquartile range; ICU, intensive care unit;

${ }^{a}$ Acute Kidney Injury, includes RIFLE classes R, I, and F.

$b_{\text {Based on data from } \mathrm{n}=99 \text { patients }}$

${ }^{c}$ Based on data from $\mathrm{n}=51$ patients 
Table IV

Predictors of 1-year Survival

\begin{tabular}{lcc}
\hline Variables & Univariate \\
& HR [95\% CI $]$ & P-value \\
\hline TEVAR approach & $0.874[0.282-2.709]$ & .815 \\
Age & $1.044[0.997-1.094]$ & .064 \\
Male Gender & $0.845[0.268-2.662]$ & .773 \\
Coronary artery disease & $1.719[0.518-5.710]$ & .376 \\
Diabetes mellitus & $0.529[0.683-4.099]$ & .542 \\
Smoking history & $1.029[0.332-3.191]$ & .960 \\
Hypertension & $0.586[0.159-2.166]$ & .423 \\
Baseline eGFR (per 10 ml/min/1.73m $\left.{ }^{2}\right)$ & $0.977[0.957-0.998]$ & .028 \\
Chronic obstructive pulmonary disease & $2.322[0.699-7.713]$ & .169 \\
Symptomatic & $1.659[0.526-5.230]$ & .387 \\
Aneurysm size & $1.229[0.778-1.940]$ & .377 \\
Acute Kidney Injury ${ }^{a}$ & $2.761[0.876-8.703]$ & .083 \\
Overall Neurologic Complication & $5.066[1.524-16.836]$ & .008 \\
CSF drain & $0.476[0.129-1.757]$ & .265 \\
\hline
\end{tabular}

Abbreviations: TEVAR, thoracic endovascular aneurysm repair; eGRF, estimated glomerular filtration rate

${ }^{a}$ Acute Kidney Injury, includes RIFLE classes R, I, and F. 